\title{
A simple method for isolation of the anteroventral periventricular and arcuate nuclei of the rat hypothalamus
}

\author{
Mohammad Saied Salehi ${ }^{1}$, Mohammad Reza Namavar ${ }^{2}$, Mohammad Reza Jafarzadeh Shirazi ${ }^{1}$, \\ Farhad Rahmanifar, Amin Tamadon ${ }^{4}$ \\ ${ }^{1}$ Department of Animal Sciences, College of Agriculture, Shiraz University, Shiraz, Iran \\ ${ }^{2}$ Histomorphometry and Stereology Research Center, Shiraz University of Medical Sciences, \& Department of Anatomical Sciences, School of Medicine, Shiraz, Iran \\ ${ }^{3}$ Division of Basic Sciences, School of Veterinary Medicine, Shiraz University, Shiraz, Iran \\ ${ }^{4}$ Division of Animal Health Management, School of Veterinary Medicine, Shiraz University and Stem cell and Transgenic Technology Research Center, \\ Shiraz University of Medical Sciences, Shiraz, Iran
}

\begin{abstract}
Objectives: The aim of the present study was to establish a simple and reliable method for separation of anteroventral periventricular (AVPV) and arcuate (Arc) nuclei in the rat hypothalamus to evaluate expression of peptide mRNAs in these nuclei using RT-PCR.

Methods: Two female Sprague -Dawley rats were perfused transcardially with $10 \%$ buffered formalin. Brains were immediately removed and diencephalon was dissected out by two coronal sections. The first section was made at rostral of optic chiasm and the second section at the caudal end of mammillary bodies. To separate the AVPV and Arc nuclei, the third section was made rostral of infundibulum which placed one part of the optic tract in rostral (anterior) division and its other part in the caudal (posterior) division. The rostral and caudal divisions were separately post-fixed and cut coronally and serially using a cryostat at 30- $\mu$ m thickness. Sections from rostral and caudal divisions of the diencephalon were stained with cresyl violet. Landmarks for Arc and AVPV nuclei were evaluated in each section.

Results: Arc and AVPV nuclei were not simultaneously detected in any stained section in rostral or caudal divisions of diencephalon.

Conclusion: By the presented method, it is easily possible to separate AVPV and Arc nuclei to evaluate the expression pattern of mRNA of peptides expressed in these two hypothalamic nuclei of the rat by using RT-PCR or other molecular methods.
\end{abstract}

Key words: anteroventral periventricular nucleus; arcuate nucleus; hypothalamus; rat

Anatomy 2012-2013;6-7:48-51, (c) 2012-2013 TSACA

\section{Introduction}

The hypothalamus is the part of the diencephalon that extends from the optic chiasma (Figure 1, OC) to the caudal border of the mammillary bodies (Figure 1, MB). It lies below the thalamus and forms the floor and the inferior part of the lateral walls of the third ventricle.
Anterior to the hypothalamus is a region, the preoptic area (POA), that is often included (from functional points of view) in the hypothalamus. Microscopically, the hypothalamus is composed of nerve cells that are arranged in groups or nuclei, many of which are not clearly segregated from one another. For purposes of

This work was financially supported by Shiraz University of Medical Sciences and Shiraz University. 
description, these nuclei are divided into periventricular, medial and lateral zones. ${ }^{[1,2]}$

The arcuate (Arc) and the anteroventral periventricular (AVPV) nuclei are the major sites in the hypothalamus that are located in its periventricular zone and involved in the control of reproduction. ${ }^{[3]}$ There is a general agreement that estrogen with positive feedback effects on AVPV neurons, causes pre-ovulatory surges of gonadotropin-releasing hormone $(\mathrm{GnRH})$ and luteinizing hormone $(\mathrm{LH}){ }^{[4]}$ Destruction of the AVPV, but not neighboring nuclei, were found to result in persistent estrous and the abolition of the estrogen-induced $\mathrm{LH}$ surge. ${ }^{[5]}$ Unlike AVPV nucleus that involved in GnRH surge, it has been reported that GnRH pulse generator has been localized to or near the Arc in rat. ${ }^{[6]}$ Kisspeptin expressing neurons that have potent stimulatory effect on GnRH cells are mainly located in the Arc and AVPV nuclei of rat hypothalamus. ${ }^{[7,8]}$ Since the activity of these neurons are regulated differently with constant amount of estrogen,${ }^{[9]}$ it is clear that for study on this neuropeptide, especially for investigation at the mRNA level with RT-PCR method, separation of these nuclei is essential.

As it is obvious, if there is a larger quantity of tissue, RNA extraction will be more applicable, and because the kisspeptin expressing neurons in the rat hypothalamus are restricted to AVPV and Arc nuclei, therefore, the aim of the present study was to set up a simple and reliable method in which by using just one cut, Arc and AVPV nuclei could be isolated into two parts.

\section{Materials and Methods}

Two adult female Sprague-Dawley rats (Rattus norvegicus) were used in the present study. The rats were anesthetized and perfused transcardially with cold $0.9 \%$ saline followed by $10 \%$ buffered formalin. Brains were immediately removed and diencephalon was dissected out by an anterior coronal section, anterior to the optic chiasma (Figure 1, AA line), and a posterior coronal cut at the posterior border of the mammillary bodies (Figure 1, BB line). For separation of the Arc from the AVPV, the third coronal cut was made in middle of the optic tract, just rostral to infundibulum (Figure 1, CC line). Then, rostral or anterior (contains AVPV nucleus) and caudal or posterior (contains Arc nucleus) divisions were post-fixed in the same fixative solution overnight and immersed in 30\% sucrose (Sigma-Aldrich Corp., St. Louis, MO, USA) in phosphate buffered saline for $48 \mathrm{~h}$ and then frozen and stored at $-20{ }^{\circ} \mathrm{C}$ until further processing. Divisions were sectioned serially and coronally using a cryostat (SLEE Medical GmbH, Mainz, Germany) at a thickness of $30 \mu \mathrm{m}$ and immersed into a

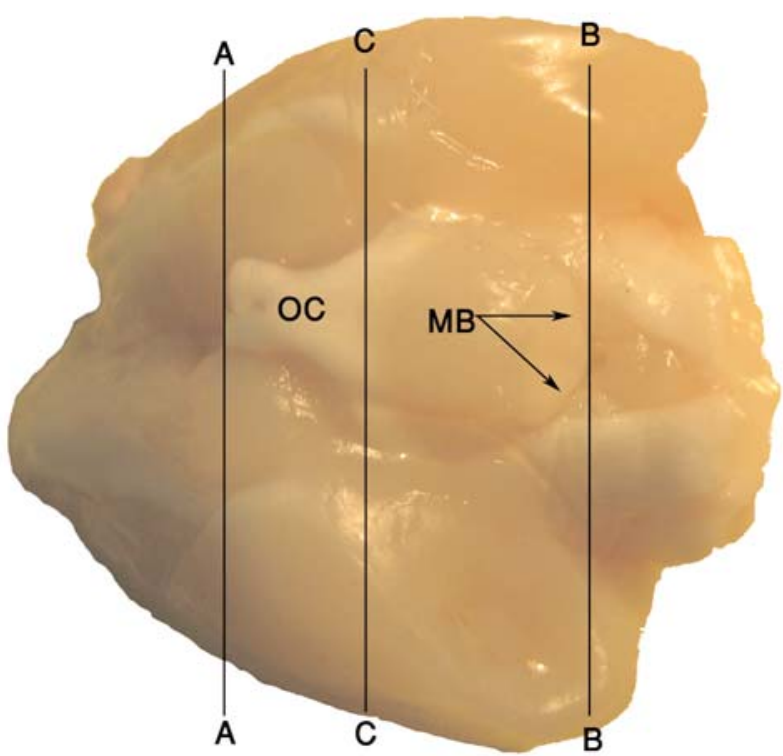

Figure 1. The ventral aspect of rat brain. Cutting sites are indicated with lines. The first coronal cut (AA) is in front of optic chiasma (OC). Second cut $(\mathrm{BB})$ is behind the mammillary body (MB). To separate the arcuate nuclei from the anteroventral periventricular nuclei (AVPV), third coronal cut (CC) was made in middle of the optic tract and in front of infundibulum.

12-well plate containing cryoprotectant solution and kept them in $-20^{\circ} \mathrm{C}$ freezer until staining. Sections from rostral and caudal divisions were slide mounted and stained with cresyl violet. Stained sections were compared with the atlas 'The Rat Brain in Stereotaxic Coordinates'. ${ }^{[10]}$ The septal nuclei, optic chiasma and anterior commissure were considered as landmarks for the AVPV nucleus (Bregma $+0.12 \mathrm{~mm}$ to Bregma -0.12 $\mathrm{mm}$ in Paxinos \& Watson atlas) and the hippocampus, fornix and mammillothalamic tracts were considered as landmarks for the Arc nucleus in each section (Bregma $1.72 \mathrm{~mm}$ to Bregma $-4.36 \mathrm{~mm}$ in Paxinos and Watson atlas). ${ }^{[10]}$ All procedures were conducted in accordance with the Guiding Principles for the Care and Use of Research Animals. Animals were sacrificed in compliance with the recommendations of Animal Care Committee for the Shiraz University of Medical Sciences (Shiraz, Iran).

\section{Results and Discussion}

Five caudal sections from the anterior division and five rostral sections from the posterior division in both brains were similar to the coronal planes (from Bregma -0.60 $\mathrm{mm}$ to Bregma $-1.44 \mathrm{~mm}$ ) in the atlas 'The Rat Brain in Stereotaxic Coordinates' by Paxinos and Watson ${ }^{[10]}$ and 
Figure 2. (a) A section of anterior division of diencephalon that shows the anteroventral periventricular nuclei (AVPV). (b) Example of rostral sections of posterior division of diencephalon that has none of the AVPV and arcuate nuclei (Arc). (c) Sections from caudal part of posterior division of diencephalon that shows Arc. Scale bar $=2$ $\mathrm{mm}$. 3v: third ventricle; ac: anterior commissure; cc: corpus callosum; fx: fornix; hippo: hippocampus; Lv: lateral ventricle; MTT: mammilothalamic tract; oc: optic chiasma; sept: septal area; SO: supraoptic nucleus; RCh: retrochiasmatic area. $\mathbf{a}, \mathbf{b}$ and $\mathbf{c}$ are similar to Bregma - $0.12 \mathrm{~mm}$, Bregma $1.32 \mathrm{~mm}$, and Bregma $-2.76 \mathrm{~mm}$, respectively, in 'The Rat Brain in Stereotaxic Coordinates' by Paxinos and Watson. ${ }^{[10]}$
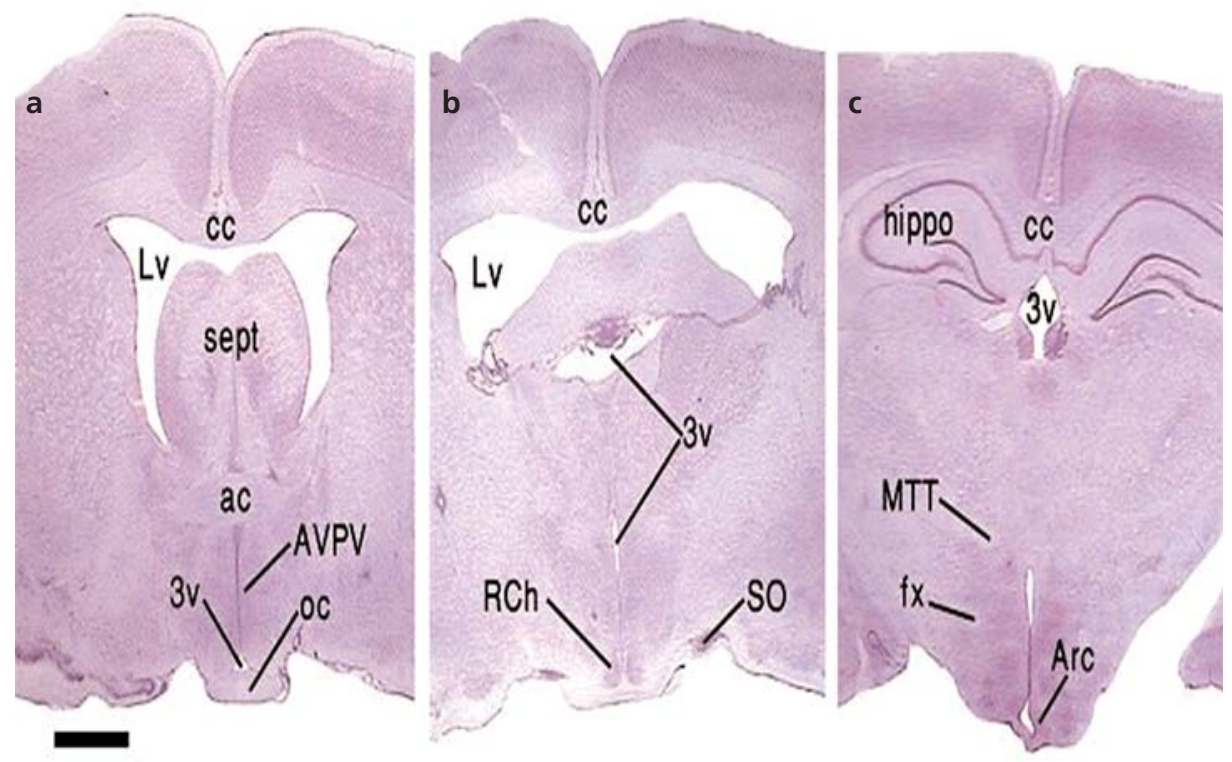

none of them had Arc or AVPV nuclei (Figure 2b). The AVPV nuclei were only found in the anterior sections and none of these sections contained Arc nuclei (Figure 2a). The Arc nuclei were found in the posterior sections, however, none of these sections contained AVPV nuclei (Figure 2c). Dissection of the hypothalamus for using in RT-PCR has been reported before; hypothalamus was dissected to the boundaries as the followings: rostral to the anterior border of the optic chiasm (Figure 1, AA), caudal to the posterior border of the mammillary body (Figure 1, BB) dorsal to the ventral border of the thalamus (Figure 3, AA), and $2 \mathrm{~mm}$ lateral to the third ventricle (Figure 3, BB) ${ }^{[1]]}$

Punch method was used for sampling of the nuclei. Bilateral punches (1 $\mathrm{mm}$ in diameter) from the medial preoptic area, which included the AVPV, were taken from Bregma +0.2 to Bregma -0.4 ; a single midline punch (1 mm diameter) was taken from Bregma -1.7 to Bregma 3.9 to include both Arc nuclei. To confirm correct punch positioning under the microscope, the punched sections were fixed with formalin and stained with crystal violet. ${ }^{[12]}$ This method was used for the investigation on KiSS-1 mRNA expression at the POA and the Arc. ${ }^{[13,14]}$ The method relatively depends on experience and skill of researcher and the quantity of tissue is also considerably small. Therefore, by using the method presented in this study, it is possible to separate the AVPV from the Arc nuclei faster and more accurately. In the present study, fixed tissues were used, but it is obvious that for determining the expression of neuropeptides that only exist in the Arc and the AVPV nuclei for real-time PCR, tissues need to be quickly dissected and frozen. ${ }^{[15]}$
We recently accomplished a research, in which we used this method for the expression of kisspeptin in Arc in lactating rats. In is study, we separated the anteroventral periventricular nucleus (AVPV) nucleus by the pre-

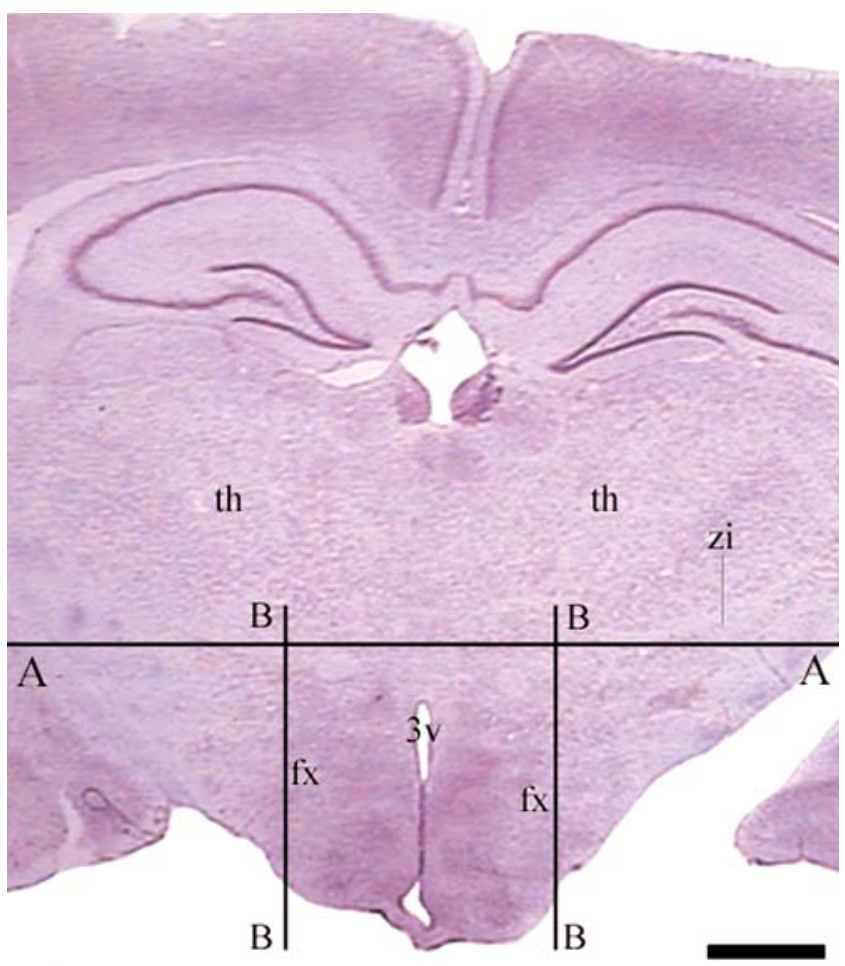

Figure 3. Coronal section of the rat diencephalon. Dorsal (AA line) and lateral (BB lines) cutting sites for dissection of the rat hypothalamus. Scale bar $=2 \mathrm{~mm}$. 3v: third ventricle; fx: fornix; th: thalamus; zi: zona incerta. 
sented method and used remaining of the hypothalamus as the Arc containing part. Our findings were similar to the previous studies that had only separated the Arc (Nourozi et al., unpublished study).

\section{Conclusion}

By the presented method it is easily possible to separate AVPV and Arc nuclei anatomically to evaluate the expression pattern of mRNA of peptides expressed in these two nuclei of hypothalamus of the rat by using RTPCR or other molecular methods.

\section{Acknowledgments}

The authors wish to thank Dr. Nader Tanideh and personnel of Animal Laboratory of Shiraz University of Medical Sciences for their kind cooperation.

\section{References}

1. Snell RS. Clinical Neuroanatomy. 7th ed. Philadelphia: Lippincott Williams \& Wilkins; 2010.

2. Saper CB. Hypothalamus. In: Paxinos G, Mai JK, eds. The Human Nervous System. 2nd ed. Amsterdam: Elsevier; 2004. p. 513-50.

3. Dhillo WS. Kisspeptin: a novel regulator of reproductive function. J Neuroendocrinol 2008;20:963-70.

4. Simerly RB. Wired for reproduction: organization and development of sexually dimorphic circuits in the mammalian forebrain. Ann Rev Neurosci 2002;25:507-36.

5. Wiegand SJ, Terasawa E, Bridson WE, Goy RW. Effects of discrete lesions of preoptic and suprachiasmatic structures in the female rat. Neuroendocrinol 1980;31:147-57.
6. Ohkura S, Tsukamura H, Maeda K. Effects of various types of hypothalamic deafferentation on luteinizing hormone pulses in ovariectomized rats. J Neuroendocrinol 1991;3:503-8.

7. Clarkson J, Herbison AE. Postnatal development of kisspeptin neurons in mouse hypothalamus; sexual dimorphism and projections to gonadotropin-releasing hormone neurons. Endocrinology 2006;147:5817-25.

8. Kauffman AS, Gottsch ML, Roa J, et al. Sexual differentiation of Kiss 1 gene expression in the brain of the rat. Endocrinology 2007; 148:1774-83.

9. Smith JT, Clifton DK, Steiner RA. Regulation of the neuroendocrine reproductive axis by kisspeptin-GPR54 signaling. Reproduction 2006;131:623-30.

10. Paxinos G, Watson C. The Rat Brain in Stereotaxic Coordinates. 6th ed. New York: Academic Press; 2007.

11. Quennell JH, Rizwan MZ, Relf HL, Anderson GM. Developmental and steroidogenic effects on the gene expression of RFamide related peptides and their receptor in the rat brain and pituitary gland. J Neuroendocrinol 2010;22:309-16.

12. Knox AM, Li XF, Kinsey-Jones JS, et al. Neonatal lipopolysaccharide exposure delays puberty and alters hypothalamic Kiss1 and Kiss1r mRNA expression in the female rat. J Neuroendocrinol 2009; 21:683-9.

13. Kinsey-Jones JS, Li XF, Knox AM, et al. Down-Regulation of hypothalamic kisspeptin and its receptor, Kiss1r, mRNA expression is associated with stress-induced suppression of luteinising hormone secretion in the female rat. J Neuroendocrinol 2009;21:20-9.

14. Yamada S, Uenoyama Y, Kinoshita M, et al. Inhibition of metastin (kisspeptin-54)-GPR54 signaling in the arcuate nucleus-median eminence region during lactation in rats. Endocrinol 2007;148: 2226-32.

15. Kim SA, Jeon JH, Son MJ, Cha J, Chun MH, Kim IB. Changes in transcript and protein levels of calbindin D28k, calretinin and parvalbumin, and numbers of neuronal populations expressing these proteins in an ischemia model of rat retina. Anat Cell Biol 2010; $43: 218-29$.

Correspondence to: Mohammad Reza Namavar, PhD Histomorphometry and Stereology Research Center, Shiraz University of Medical Sciences, Shiraz, Iran Phone: +98-711-2304372

e-mail: namavarreza@yahoo.com, namavarm@sums.ac.ir

Conflict of interest statement: No conflicts declared. 